\title{
SOME ISOMORPHISMS OF ABELIAN GROUPS INVOLVING THE TOR FUNCTOR
}

\author{
PATRICK KEEF
}

(Communicated by Warren J. Wong)

\begin{abstract}
Given a reduced group $G$, the class of groups $A$ such that $A \cong$ $\operatorname{Tor}(A, G)$ is studied. A complete characterization is obtained when $G$ is separable.
\end{abstract}

\section{INTRODUCTION}

In this note, the term "group" will mean an abelian $p$-group for some fixed prime $p$. The notation and terminology will generally follow [3]. By a $\oplus_{c}$ we mean a direct sum of cyclic groups.

An important result in the study of the Tor functor states that for any group $A$ there is an isomorphism $A \cong \operatorname{Tor}\left(A, Z_{p^{\infty}}\right)$. The purpose of this work is to explore the class of groups that result when $Z_{p^{\infty}}$ is replaced by some other group, i.e., given a group $G$, find all groups $A$ such that $A \cong \operatorname{Tor}(A, G)$. We are primarily concerned with reduced $G$. Examples of groups $A$ satisfying $A \cong \operatorname{Tor}(A, G)$ are found of any length not exceeding the length of $G$. We also construct examples of groups $A$ that are Tor-idempotent, i.e. $A \cong \operatorname{Tor}(A, A)$.

Our most impressive results occur in the case of separable groups (i.e., groups with no elements of infinite height). A new characterization of $\oplus_{c}$ is given in terms of iterated torsion products of separable groups. This allows us to conclude that when $G$ is separable, any group $A$ satisfying $A \cong \operatorname{Tor}(A, G)$ is a $\oplus_{c}$ and hence that any separable Tor-idempotent group is a $\oplus_{c}$.

\section{BASIC PROPERTIES}

We begin this section with a review of some standard ideas. If $\alpha$ is an ordinal, a short exact sequence $0 \rightarrow C \rightarrow B \rightarrow A \rightarrow 0$ is $p^{\alpha}$-pure if it represents an element of $p^{\alpha} \operatorname{Ext}(A, C)$. If this group of extensions is trivial for every $C$ then $A$ is $p^{\alpha}$-projective. We assume the standard facts on $p^{\alpha}$-purity that can be found, for example, in [5]. In particular, $p^{\omega}$-purity is regular purity, and a $p^{\omega}$-projective is a $\oplus_{c}$.

Received by the editors July 14, 1989.

1980 Mathematics Subject Classification (1985 Revision). Primary 20K10, 20K40.

Key words and phrases. Abelian p-group, Tor functor, separable groups, direct sums of cyclic groups. 
The following facts on the Tor functor are due to Nunke [13, 14].

Lemma 1.1. Suppose $A, B, C$, and $G$ are groups, and $\alpha$ is an ordinal.

(a) $p^{\alpha} \operatorname{Tor}(A, G) \cong \operatorname{Tor}\left(p^{\alpha} A, p^{\alpha} G\right)$.

(b) $f_{\operatorname{Tor}(A, G)}(\alpha)=f_{A}(\alpha) f_{G}(\alpha)+f_{A}(\alpha) r\left(p^{\alpha+1} G\right)+r\left(p^{\alpha+1} A\right) f_{G}(\alpha)$ (where $f_{X}$ and $r(X)$ denote the Ulm function and rank of $X$ respectively.)

(c) If $G$ is $p^{\alpha}$-projective, so is $\operatorname{Tor}(A, G)$.

(d) If $\alpha$ is an infinite ordinal and $0 \rightarrow C \rightarrow B \rightarrow A \rightarrow 0$ is $p^{\alpha}$-pure, so is

$$
0 \rightarrow \operatorname{Tor}(C, G) \rightarrow \operatorname{Tor}(B, G) \rightarrow \operatorname{Tor}(A, G) \rightarrow 0 .
$$

(e) If $R_{1}, R_{2}$ and $S_{1}, S_{2}$ are subgroups of $A$ and $B$ respectively, then

$$
\operatorname{Tor}\left(R_{1}, S_{1}\right) \cap \operatorname{Tor}\left(R_{2}, S_{2}\right) \cong \operatorname{Tor}\left(R_{1} \cap R_{2}, S_{1} \cap S_{2}\right) \text {. }
$$

If $G$ is a group, let $\mathscr{T}_{G}$ denote the class of groups $A$ for which $A \cong$ $\operatorname{Tor}(A, G)$.

Proposition 1.2. For any group $G$, the class $\mathscr{T}_{G}$ contains nonzero, reduced groups, in fact, it contains groups that are $\oplus_{c}$.

Proof. If $A$ is a $\oplus_{c}$ with sufficiently large Ulm invariants, it can be seen from Lemma $1.1(\mathrm{~b})$ that $f_{A}(n)=f_{\operatorname{Tor}(A, G)}(n)$ for every $n<\omega$. Since $\operatorname{Tor}(A, G)$ will also be a $\oplus_{c}$, the result is proven.

The classes $\mathscr{T}_{G}$ are clearly closed under direct sums. Only under special circumstances, however, are they closed under summands. Observe

$$
\mathscr{T}_{Z_{p^{n}}}=\left\{p^{n}-\text { bounded groups }\right\}, \mathscr{T}_{Z_{p}}=\{\text { abelian groups }\},
$$

and these classes are closed with respect to summands. They are the only ones.

Proposition 1.3. If $G$ is a group, $\mathscr{T}_{G}$ is closed with respect to summands iff $G$ is cocyclic.

Proof. Suppose $\mathscr{T}_{G}$ is closed with respect to summands. By Proposition 1.2, there is a nonzero $\oplus_{c}$ group $A$ in $\mathscr{T}_{G}$. If $A^{\prime}$ is a cyclic summand of $A$, say of order $p^{n}$, then $A^{\prime} \cong \operatorname{Tor}\left(A^{\prime}, G\right) \cong G\left[p^{n}\right]$. So $G\left[p^{n}\right]$ is cyclic and $G$ is cocyclic.

Recall that a group is said to be starred if it has the same cardinality as its basic subgroups and fully starred if every subgroup is starred. The importance of this notion for the present investigation is contained in the following:

Proposition 1.4. If $G$ is reduced and $A$ is in $\mathscr{T}_{G}$, then $A$ is reduced and fully starred.

Proof. If $\alpha$ is the length of $G$, then by Lemma $1.1(\mathrm{a}), p^{\alpha} A \cong \operatorname{Tor}\left(p^{\alpha} A, p^{\alpha} G\right)$ $=0$, so $A$ is reduced. By Corollary 2.5 of [13], $\operatorname{Tor}(A, G) \cong A$ must be fully starred.

The last proposition shows, for example, that an unbounded torsion complete group is not in $\mathscr{T}_{G}$ for any reduced $G$. In another direction, only under certain 
circumstances can totally projective groups, or even $S$-groups, be in $\mathscr{T}_{G}$ (see [15] for the definition of an $S$-group).

Proposition 1.5. If $G$ is reduced and $A$ in an $S$-group in $\mathscr{T}_{G}$, then $A$ is a dsc (i.e., a direct sum of countable groups).

Proof. By Proposition 1.4, $A$ must be reduced. By [16, Corollary 3.6], an $S$-group of the form $\operatorname{Tor}(A, G) \cong A$ must be totally projective, and by [6, Corollary 4], a totally projective group of this form must be a disc.

A group $G$ is $C$-decomposable iff it has a summand which is a $\bigoplus_{c}$ of the same final rank as $G$ itself. In [4] it was shown that when $X$ and $Y$ are reduced groups, $\operatorname{Tor}(X, Y)$ is $C$-decomposable. We wish to examine the $\bigoplus_{c}$ summands that $\operatorname{Tor}(X, Y)$ admits a bit more closely.

We say that a group $G$ is $B$-decomposable if $G$ has a summand which is isomorphic to a basic subgroup of $G$. In particular, if $G$ is $B$-decomposable and $f_{G}(n)$ is infinite or 0 for all $n<\omega$, then $G \cong A \oplus B$, with $B$ isomorphic to a basic subgroup of $G$, and the Ulm invariants of $B$ are also infinite or 0 . Therefore, $B \cong B \oplus B$, and hence $G \cong A \oplus B \cong A \oplus B \oplus B \cong G \oplus B$.

In general, $B$ - and $C$-decomposability are independent notions. If $A=$ $\oplus_{n<\omega} Z_{p^{n+1}}$, then

$$
\bar{A} \oplus\left(\oplus_{\aleph_{0}} A\right)
$$

is clearly $B$-decomposable, but not $C$-decomposable. On the other hand, if $X=\oplus_{n<\omega} Z_{p^{2 n+1}}$ and $Y=\oplus_{n<\omega} Z_{p^{2 n+2}}$, then

$$
\bar{X} \oplus\left(\oplus_{2{ }^{\aleph_{0}}} Y\right)
$$

is clearly $C$-decomposable, but not $B$-decomposable (these assertions can be checked using standard facts on direct sums of torsion complete groups; see [3, $\S 73])$. In a positive direction we have:

Proposition 1.6. If $G$ is a fully starred and B-decomposable, then $G$ is $C$ decomposable.

Proof. Suppose $B$ is a basic subgroup of $G$ and $r\left(p^{n} G\right)$ equals the final rank of $G$. For every $m \geq n, p^{m} B$ is a basic subgroup of $p^{m} G$, and since $G$ is fully starred, $r\left(p^{m} B\right)=r\left(p^{m} G\right)=r\left(p^{n} G\right)$. Therefore, $G$ and $B$ have the same final rank. Since $G$ has a summand isomorphic to $B, G$ is $C$-decomposable.

Our interest in $B$-decomposability stems from the following result.

Theorem 1.7. If $A$ and $G$ are reduced groups, then $\operatorname{Tor}(A, G)$ is B-decomposable.

Proof. Clearly the direct sum of $B$-decomposable groups is $B$-decomposable and any $\oplus_{c}$ is $B$-decomposable. First, $A \cong A_{1} \oplus A_{2}$ where $A_{2}$ is bounded, and $r\left(A_{1}\right)$ equals the final rank of $A$. Since $\operatorname{Tor}(A, G) \cong \operatorname{Tor}\left(A_{1}, G\right) \oplus \operatorname{Tor}\left(A_{2}, G\right)$, and $\operatorname{Tor}\left(A_{2}, G\right)$ is bounded and hence a $\oplus_{c}$, we may assume that the rank of $A$ is infinite and equals its final rank. We can clearly make the same assumptions 
about $G$. Let $A^{\prime}$ be a lower basic subgroup of $A$ (so $\left.r\left(A / A^{\prime}\right)=r(A)\right)$. There is a pure short exact sequence

$$
0 \rightarrow \operatorname{Tor}\left(A^{\prime}, G\right) \rightarrow \operatorname{Tor}(A, G) \rightarrow K \rightarrow 0,
$$

where

$$
K=\oplus_{r(A)} G \cong \oplus_{n<\omega}\left(\oplus_{r(A)} G\right)
$$

For $n<\omega$,

$$
\oplus_{r(A)} G=\left(\oplus_{r(A)} G\right) \oplus\left(\oplus_{r(A) f_{G}(n)} Z_{p^{n+1}}\right)
$$

so if $L=\oplus_{n<\omega}\left(\oplus_{r(A) f_{G}(n)} Z_{p^{n+1}}\right)$, it follows that $K$ has a summand isomorphic to $L$. The purity of $(*)$ easily implies that we can "pull this back" to a summand of $\operatorname{Tor}(A, G)$ isomorphic to $L$. Since $L \cong L \oplus L$,

$$
\operatorname{Tor}(A, G) \cong \operatorname{Tor}(A, G) \oplus\left(\oplus_{n<\omega}\left(\oplus_{r(A) f_{G}(n)} Z_{p^{n+1}}\right)\right) .
$$

Similarly,

$$
\operatorname{Tor}(A, G) \cong \operatorname{Tor}(A, G) \oplus\left(\oplus_{n<\omega}\left(\oplus_{f_{A}(n) r(G)} Z_{p^{n+1}}\right)\right),
$$

from which it follows that $\operatorname{Tor}(A, G)$ has a summand isomorphic to,

$$
\oplus_{n<\omega}\left(\oplus_{f_{A}(n) r(G)+r(A) f_{G}(n)} Z_{p^{n+1}}\right)
$$

Since $r(G) \geq f_{G}(n)$ and $r(A) \geq f_{A}(n)$.

$$
f_{A}(n) r(G)+r(A) f_{G}(n)=f_{A}(n) f_{G}(n)+f_{A}(n) r(G)+r(A) f_{G}(n),
$$

and the theorem follows from Lemma 1.1(b).

Corollary 1.8. If $G$ is reduced and $A$ is in $\mathscr{T}_{G}$, then $A$ is $B$-and $C$-decomposable.

Proof. By Proposition 1.4, $A$ will also be reduced, so the result follows from Propositions 1.6 and 1.7.

We end this section with a computation. In [12] the generalized Prüfer group, $H_{\alpha}$ was defined for each ordinal $\alpha$. If $\alpha$ is infinite, there is a $p^{\alpha}$-pure exact sequence

$$
0 \rightarrow M_{\alpha} \rightarrow H_{\alpha} \rightarrow Z_{p^{\infty}} \rightarrow 0 .
$$

For any group $A$ a second $p^{\alpha}$-pure exact sequence,

$$
0 \rightarrow \operatorname{Tor}\left(A, M_{\alpha}\right) \rightarrow \operatorname{Tor}\left(A, H_{\alpha}\right) \rightarrow A \rightarrow 0
$$

results. It follows that $A$ is $p^{\alpha}$-projective iff it is a summand of $\operatorname{Tor}\left(A, H_{\alpha}\right)$. This leads to the question: When is it true that these two groups are actually isomorphic, i.e., when is $A \in \mathscr{T}_{H_{\alpha}}$ ? If $\alpha$ is finite, $H_{\alpha}$ is cocyclic and $\mathscr{T}_{H_{\alpha}}$ consists of the $p^{\alpha}$-bounded groups. Although we do not answer the question for arbitrary ordinals, we can handle the important case $\alpha=\omega+n$. 
Theorem 1.9. Suppose $n<\omega$ and $A$ is unbounded. Then $A$ is in $\mathscr{T}_{H_{\omega+n}}$ iff $A$ is $p^{\omega+n}$-projective, B-decomposable and for $m<\omega, f_{A}(m)=r\left(p^{m} A\right)$.

Proof. Since for $m<\omega, f_{H_{\omega+n}}(m)=1$ and $r\left(p^{m+1} H_{\omega+n}\right)=\aleph_{0}$, we have

$$
f_{\operatorname{Tor}\left(A, H_{\omega+n}\right)}(m)=f_{A}(m) \cdot 1+r\left(p^{m+1} A\right) \cdot 1+f_{A}(m) \cdot \aleph_{0}=r\left(p^{m} A\right) .
$$

Suppose first that $A \in \mathscr{T}_{H_{\omega+n}}$. By Lemma 1.1(c), $A$ is $p^{\omega+n}$-projective. By Corollary 1.8, $A$ must be $B$-decomposable. Since $f_{A}(m)=f_{\operatorname{Tor}\left(A, H_{\omega+n}\right)}(m)=$ $r\left(p^{m} A\right)$, we are done.

To establish the converse, note that $M_{\omega+n} \cong \oplus_{m<\omega} Z_{p^{m+1}} \cong H_{\omega}$. In particular, $\operatorname{Tor}\left(A, M_{\omega+n}\right)$ is a $\oplus_{c}$. For every $m<\omega$ we have,

$$
f_{A}(m)=r\left(p^{m} A\right)=f_{\operatorname{Tor}\left(A, M_{\omega+n}\right)}(m) \text {. }
$$

So $\operatorname{Tor}\left(A, M_{\omega+n}\right)$ is isomorphic to a basic subgroup of $A$. As $A$ is $B$ decomposable and $f_{A}(m)$ is infinite for $m<\omega$, we have $A \cong A \oplus \operatorname{Tor}\left(A, M_{\omega+n}\right)$. Since $A$ is $p^{\omega+n}$-projective,

$$
\operatorname{Tor}\left(A, H_{\omega+n}\right) \cong A \oplus \operatorname{Tor}\left(A, M_{\omega+n}\right) \cong A,
$$

which proves the result.

Because bounded groups are $\oplus_{c}$, it is straightforward, but slightly tedious, to extend the last result to all $p^{\omega+n}$-projective groups by applying Lemma 1.1(b).

\section{EXAMPLES}

In this section we construct some examples. We will use extensively the iterated torsion product $\operatorname{Tor}\left(A_{1}, \ldots, A_{n}\right)$. This product is both commutative and associative. To simplify our notation, we write this product multiplicatively, i.e., $A_{1} A_{2} \cdots A_{n}$. In particular, $A^{n}=\operatorname{Tor}(A, \ldots, A) \quad(n$-copies $)$ and $A^{1}=A$. Define $A^{0}=Z_{p^{\infty}}$ (this makes sense because $Z_{p^{\infty}}$ acts as an identity for Tor). For a family of groups $\left\{A_{i}\right\}_{i \in I}$ and a group $B$, we have $\oplus_{i}\left(A_{i} B\right) \cong\left(\oplus_{i} A_{i}\right) B$, which we denote simply by $\oplus_{i} A_{i} B$. Our examples will use the following simple observation:

Lemma 2.1. Suppose $\rho$ is an infinite cardinal, $\alpha$ is an infinite ordinal, and $X$ is a reduced group such that $\rho \leq \min _{\delta<\alpha} r\left(p^{\delta} X\right)$. If $H$ is $p^{\alpha}$-projective, then $X H \cong\left(\oplus_{\rho} H\right) \oplus X H$.

Proof. Suppose $X^{\prime}$ is a $p^{\alpha}$-pure subgroup of $X$ such that $X / X^{\prime}$ is divisible of rank $\rho$ (if $\alpha$ is isolated we can choose $X^{\prime}$ to be a $p^{\alpha-1}$-high subgroup, and if $\alpha$ is a limit such an $X^{\prime}$ exists by [1]). There is, therefore, a $p^{\alpha}$-pure exact sequence,

which must split. So,

$$
0 \rightarrow X^{\prime} H \rightarrow X H \rightarrow \oplus_{\rho} H \rightarrow 0
$$

$$
X H \cong\left(\oplus_{\rho} H\right) \oplus X^{\prime} H \cong\left(\oplus_{\rho} H\right) \oplus\left(\oplus_{\rho} H\right) \oplus X^{\prime} H \cong\left(\oplus_{\rho} H\right) \oplus X H .
$$


If $G$ is a reduced group of length $\alpha$, by Lemma $1.1(\mathrm{a})$ a group in $\mathscr{T}_{G}$ can have length at most $\alpha$. The following is a converse to this statement.

Theorem 2.2. Suppose $G$ is a reduced group of length $\alpha$ and $\beta \leq \alpha$. Then there is an $A$ in $\mathscr{T}_{G}$ of length $\beta$.

Proof. We may clearly assume $\alpha$ is infinite. Suppose $H$ is a $p^{\alpha}$-projective of length $\beta\left(H_{\beta}\right.$, for example). For every finite ordinal $n$ define $X_{n}=\oplus_{\aleph_{0}} G^{n}$ (so $X_{0} \cong \oplus_{\aleph_{0}} Z_{p^{\infty}}$ ). Let $A=\oplus_{n<\omega} X_{n} H$. Clearly $A$ has length $\beta$ and

$$
A G \cong \oplus_{n<\omega}\left(\oplus_{\aleph_{0}} G^{n+1}\right) H \cong \oplus_{n<\omega} X_{n+1} H .
$$

If $\delta<\alpha$, then $\aleph_{0} \leq r\left(p^{\delta} X_{1}\right)$, so by Lemma 2.1,

$A G \cong X_{1} H \oplus\left(\oplus_{1<n<\omega} X_{n} H\right) \cong\left(\oplus_{\aleph_{0}} H\right) \oplus X_{1} H \oplus\left(\oplus_{1<n<\omega} X_{n} H\right) \cong \oplus_{n<\omega} X_{n} H=A$,

which proves the result.

Corollary 2.3. If $G$ is a reduced IT group (i.e., $G$ is isomorphic to an isotype subgroup of a totally projective group), and $\beta \leq \alpha$, we can find an $A$ in $\mathscr{T}_{G}$ which is an IT group of length $\beta$.

Proof. By [9, Corollary 6], the torsion product of IT groups is once again an IT group. So if $G$ is an IT group and $H=H_{\beta}$, the group $A$ constructed in Theorem 2.2 will be an IT group.

Recall, we said a group $A$ is Tor-idempotent if $A \cong \operatorname{Tor}(A, A)$, i.e., if $A$ is in $\mathscr{T}_{A}$.

In [8] it was noticed that there are examples of Tor-idempotent groups in the dsc groups. However, we have the following limitation on this class:

Corollary 2.4. An S-group that is Tor-idempotent is a dsc.

Proof. This follows from Proposition 1.5.

In a positive direction, in [11], it was noted that if $A=\oplus_{\aleph_{1}} H_{\omega_{1}+1}, A^{2}$ is an $A$-group (see [7] for a definition of this term) and $A G \cong A^{2} G$ for every reduced totally projective group $G$. Therefore, $A^{2} \cong A^{3} \cong A^{4}$, so $A^{2}$ is a Tor-idempotent group of length $\omega_{1}+1$. More generally we have,

Theorem 2.5. There are reduced Tor-idempotent groups of arbitrary length. In fact, there are examples which are IT groups.

Proof. Let $\alpha$ be an ordinal, and $H$ a reduced totally projective group of length $\alpha$ whose Ulm invariants are 0 or infinite. For integers $j \geq 1$,

$$
H^{j} \oplus H^{j} \cong H H^{j-1} \oplus H H^{j-1} \cong(H \oplus H) H^{j-1} \cong H H^{j-1} \cong H^{j} .
$$

Let $A=\oplus_{0<n<\omega} H^{n}$. By Lemma 1.1(a), $A$ has length $\alpha$. Since the torsion product of IT groups is once again an IT group, $A$ is an IT group. Note

$$
A^{2} \cong\left(\oplus_{0<m<\omega} H^{m}\right)\left(\oplus_{0<n<\omega} H^{n}\right) \cong \oplus_{2 \leq j<\omega}\left(\oplus_{m+n=j} H^{j}\right) \cong \oplus_{2 \leq j<\omega} H^{j} .
$$


Applying Lemma 2.1 we have

$$
A^{2} \cong H^{2} \oplus\left(\oplus_{3 \leq j<\omega} H^{j}\right) \cong\left(\oplus_{\aleph_{0}} H\right) \oplus H^{2} \oplus\left(\oplus_{3 \leq j<\omega} H^{j}\right) \cong \oplus_{0<j<\omega} H^{j}=A \text {, }
$$

which proves the result.

\section{SePARABLE GROUPS}

In this section we concentrate on separable groups. Before entering into our main discussion, we note the following consequence of Shelah's singular compactness theorem.

Lemma 3.1. Suppose $\alpha$ is a singular cardinal and $A$ is a group of cardinality $\alpha$. If every subgroup of $A$ of strictly smaller cardinality is $a \oplus_{c}$ (i.e., $A$ is an $\alpha-\oplus_{c}$ ) then $A$ is $a \oplus_{c}$.

Proof. This can easily be observed by considering the socle as a valuated vector space. If $S$ is a subsocle of $A$ and $|S|<\alpha$, then $S$ must be contained in a pure subgroup $B$ of $A$ with $|B|<\alpha$. Hence $B$ is a $\oplus_{c}$ and $B[p]$ is a free valuated vector space containing $S$. The result follows from the main theorem of [2].

We separate out the following technical observation for later use.

Lemma 3.2. If $Y_{1}, \ldots, Y_{m}$ are subgroups of $X_{1}, \ldots, X_{m}$ respectively, there is a left exact sequence

$$
0 \rightarrow Y_{1} \cdots Y_{m} \rightarrow X_{1} \cdots X_{m} \rightarrow \oplus_{i=1}^{m} X_{1} \cdots X_{i-1}\left(X_{i} / Y_{i}\right) X_{i+1} \cdots X_{m}
$$

Proof. The right map is the obvious one (the diagonal of the natural maps). The kernel of this is clearly

$$
\bigcap_{i=1}^{m} X_{1} \cdots X_{i-1} Y_{i} X_{i+1} \cdots X_{m} \cong Y_{1} \cdots Y_{m},
$$

where this last isomorphism results from the obvious generalization of Lemma 1.1 (e) to iterated torsion products.

For any positive integer $n$ we define a class $\mathscr{S}_{n}$ of separable groups as follows: a group $A$ is in $\mathscr{S}_{n}$ iff there are separable groups $X_{1}, \ldots, X_{n}$ such that $A$ is isomorphic to a subgroup of $X_{1} \cdots X_{n}$. Clearly $\mathscr{S}_{1}$ is the class of all separable groups. We collect a few elementary properties of these classes in the following:

Lemma 3.3. Suppose $n$ is a positive integer.

(a) $\mathscr{S}_{n} \supseteq \mathscr{S}_{n+1}$.

(b) The class $\mathscr{S}_{n}$ is closed with respect to subgroups and direct sums.

(c) If $A$ is in $\mathscr{S}_{n}$ and $G$ is any group (possibly nonseparable), then $A G$ is in $\mathscr{S}_{n}$.

(d) If $A$ is in $\mathscr{S}_{n}$ and $G$ is in $\mathscr{S}_{m}$, then $A G$ is in $\mathscr{S}_{n+m}$.

(e) $A$ is in $\mathscr{S}_{n}$ iff there is a torsion complete group $\bar{B}$ such that $A$ is isomorphic to a subgroup of $\bar{B}^{n}$. 
Proof. (a) If $A$ is in $\mathscr{S}_{n+1}$ there is an injection.

$$
A \rightarrow X_{1} \cdots X_{n} X_{n+1} \cong X_{1} \cdots X_{n-1}\left(X_{n} X_{n+1}\right)
$$

so $A$ is in $\mathscr{S}_{n}$.

(b) That $\mathscr{S}_{n}$ is closed with respect to subgroups is trivial. As to direct sums, if for $i \in I, A_{i}$ is isomorphic to a subgroup of $X_{i, 1} \cdots X_{i, n}$, then $\oplus_{i \in I} A_{i}$, is clearly isomorphic to a subgroup of $\left(\oplus_{i \in I} X_{i, 1}\right) \cdots\left(\oplus_{i \in I} X_{i, n}\right)$.

(c) If $A \rightarrow X_{1} \cdots X_{n}$ is an injection, then

$$
A G \rightarrow X_{1} \cdots X_{n} G \cong X_{1} \cdots X_{n-1}\left(X_{n} G\right)
$$

is an injection and $A G$ is in $\mathscr{S}_{n}$.

(d) If $A \rightarrow X_{1} \cdots X_{n}$ and $G \rightarrow Y_{1} \cdots Y_{m}$ are injections, then there is an induced map

$$
A G \rightarrow X_{1} \cdots X_{n} Y_{1} \cdots Y_{m}
$$

which is clearly an injection.

(e) If $A \rightarrow X_{1} \cdots X_{n}$ is an injection and $\bar{B}$ is a torsion complete group containing isomorphic copies of $X_{1}, \ldots, X_{n}$, then $A$ is clearly isomorphic to a subgroup of $\bar{B}^{n}$.

Define $\mathscr{S}_{\infty}=\bigcap_{n=1}^{\infty} \mathscr{S}_{n}$. Clearly Lemma $3.2(\mathrm{~b})$ and (c) remain valid if $n$ is replaced by $\infty$. Our next result gives a rather surprising characterization of the groups in $\mathscr{S}_{\infty}$.

Theorem 3.4. $A$ group $A$ is in $\mathscr{S}_{\infty}$ iff $A$ is $a \oplus_{c}$.

Proof. Suppose $A$ is a $\oplus_{c}$. If $C$ is a cyclic group, for every positive integer $n, C \cong C^{n}$, so $C$ is in $\mathscr{S}_{\infty}$. Since $\mathscr{S}_{\infty}$ is closed with respect to direct sums, $A$ is in $\mathscr{S}_{\infty}$.

Conversely, suppose $A$ is in $\mathscr{S}_{\infty}$. We prove the result by inducting on the cardinality of $A$ which we denote by $\alpha$. If $\alpha \leq \aleph_{0}$ the result is clear since any countable separable group is a $\oplus_{c}$. Assume now that $\alpha$ is uncountable and the result is valid for all groups of strictly smaller cardinality.

Case I. $\alpha$ is singular. Note every subgroup of $A$ is in $\mathscr{S}_{\infty}$, so by induction, every subgroup of strictly smaller cardinality is a $\oplus_{c}$. By Lemma 3.1, this case is established.

Case II. $\alpha$ is regular. Construct a filtration $\left\{A_{i}\right\}_{i<\alpha}$ of $A$ consisting of pure subgroups. If $n$ is a positive integer there are separable groups $X_{1}, \ldots, X_{n+1}$ such that $A$ can be identified with a subgroup of $X_{1} \cdots X_{n+1}$. There is no loss of generality in assuming that all the $X$ 's have cardinality $\alpha$. By a standard "back-and-forth" argument (easy, but rather messy to write down), there is a cub (closed and unbounded) $C_{n} \subseteq \alpha$ and filtrations $\left\{X_{1, i}\right\}_{i<\alpha}, \ldots,\left\{X_{n+1, i}\right\}_{i<\alpha}$ of $X_{1}, \ldots, X_{n+1}$ respectively, such that for all $i \in C_{n}$,

$$
A_{i}=A \cap\left(X_{1, i} \cdots X_{n+1, i}\right) \text {. }
$$


If $i$ and $j$ are in $C_{n}$ with $i<j$, then using Lemma 2.1, the above equation and some easy diagram chasing, there is a diagram

where

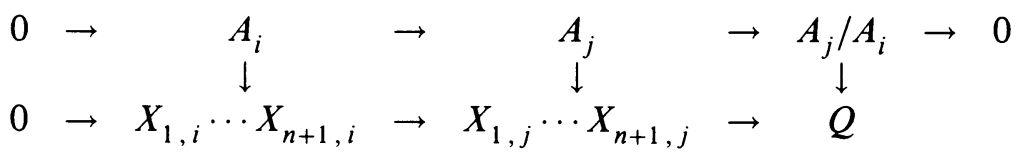

$$
Q=\bigoplus_{k=1}^{n+1} X_{1, j} \cdots X_{k-1, j}\left(X_{k, j} / X_{k, i}\right) X_{k+1, j} \cdots X_{n+1, j}
$$

whose vertical arrows are injective. By Lemma 3.3(b) and (c), $Q$ is in $\mathscr{S}_{n}$, so $A_{j} / A_{i}$ is in $\mathscr{S}_{n}$, also.

Let $C=\bigcap_{n=1}^{\infty} C_{n}$. So $C$ is also a cub in $\alpha$ and for all $i, j$ in $C$ with $i<j, A_{j} / A_{i}$ is in $\bigcap_{n=1}^{\infty} \mathscr{S}_{n}=\mathscr{S}_{\infty}$. By induction, $A_{j} / A_{i}$ is a $\oplus_{c}$. Suppose $c: \alpha \rightarrow C$ is a continuous bijection. Since each $A_{i}$ is a pure subgroup, the short exact sequence

$$
0 \rightarrow A_{c(i)} \rightarrow A_{c(i+1)} \rightarrow A_{c(i+1)} / A_{c(i)} \rightarrow 0
$$

splits, implying that $A \cong \oplus_{i \in \alpha} A_{c(i+1)} / A_{c(i)}$ is a $\oplus_{c}$.

Corollary 3.5. Suppose $G$ is a separable group and $A$ is in $\mathscr{T}_{G}$. Then $A$ is a $\oplus_{c}$.

Proof. Clearly $A$ is separable. If $A$ is in $\mathscr{S}_{n}$, then since $G$ is in $\mathscr{S}_{1}$, by Lemma 3.3(d), $A \cong A G$ will be in $\mathscr{S}_{n+1}$. Therefore, $A$ must be in $\mathscr{S}_{\infty}$, i.e., it is a $\oplus_{c}$.

Corollary 3.6. If $A$ is a separable Tor-idempotent group, then $A$ is $a \oplus_{c}$.

Proof. This follows from the last Corollary, since $A$ being Tor-idempotent means $A$ is in $\mathscr{T}_{A}$.

\section{MORE EXAMPLES}

We conclude with some examples which illuminate the results of $\S 3$.

Example 1. If $G$ is reduced, but not separable, there may be separable $A$ in $\mathscr{T}_{G}$ which are not $\oplus_{c}$. For example, if $G=H_{\omega+n}$, many examples can be constructed using Theorem 1.9.

Example 2. For separable $G$, Corollary 3.5 implies that the class $\mathscr{T}_{G}$ depends only on the cardinals $f_{G}(n), r\left(p^{n} G\right)$ and not on the particular structure of $G$ at all. In fact, $\mathscr{T}_{G}$ consists of exactly those $\oplus_{c}$ whose Ulm functions satisfy Lemma 1.1(b). This fails to be the case when $G$ is reduced, but not separable. For example, suppose $G \cong \oplus_{2^{\mathrm{N}_{0}}} H_{\omega+1}, B \cong \oplus_{n<\omega} Z_{p^{n+1}}$, and $G^{\prime}=G \oplus \bar{B}$. Clearly $f_{G}(\alpha)=f_{G^{\prime}}(\alpha)$ and $r\left(p^{\alpha} G\right)=r\left(p^{\alpha} G^{\prime}\right)$ for every ordinal $\alpha$. Since $G$ is a dsc, so is $\operatorname{Tor}(G, G)$, and it can easily be seen that they have the same Ulm function. Therefore, $G$ is in $\mathscr{T}_{G}$. However, $G$ is not in $\mathscr{T}_{G^{\prime}}$, since $\operatorname{Tor}\left(G, G^{\prime}\right)$ has a summand $\operatorname{Tor}(G, \bar{B})$ which is not a dsc (see [14, Theorem 6]). 
Example 3. By Lemma 3.3(a) the classes $\mathscr{S}_{n}$ form a descending chain. To see that they are distinct we can proceed as follows. If $m$ is a positive integer, $X$ is a separable group of cardinality $\aleph_{m}$ and $\left\{Y_{i}\right\}_{i<\aleph_{m}}$ is a filtration of $X$, let

$$
\Gamma_{m}^{\prime} X=\left\{i: p^{\omega}\left(X / Y_{i}\right) \neq 0\right\} \subseteq \aleph_{m}
$$

and $\Gamma_{m} X$ be the image of $\Gamma_{m}^{\prime} X$ in the usual quotient boolean algebra. It is straightforward to construct a separable group $X_{m}$ such that $\Gamma_{m} X_{m} \neq 0$. Clearly $A=X_{1} X_{2} \cdots X_{n}$ is in $\mathscr{S}_{n}$. If $A$ were in $\mathscr{S}_{n+1}$, then $A$ would be a subgroup of $Y_{1} \cdots Y_{n+1}$ for some separable $Y$ 's. Clearly we may assume that each $Y$ has cardinality at most $\aleph_{n}$. By [10, Theorem 5] $Y_{1} \cdots Y_{n+1}$ is a $\oplus_{c}$. However, by [10, Theorem 7] $A$ is not a $\oplus_{c}$. This contradiction implies that $A$ is in $\mathscr{S}_{n}$, but not in $\mathscr{S}_{n+1}$.

For a separable group $X$, let $\mu(X)$ denote the largest $n=1,2, \ldots, \infty$ such that $X$ is in $\mathscr{S}_{n}$. Our last example constructs for each positive integer $n$ a group $A$ with $\mu(A)=n$. Observe that if $Y$ is a subgroup of $X$, we have $\mu(X) \leq \mu(Y)$, i.e., $\mu$ reverses orderings. Because of Lemma 3.3(e) and the importance of torsion complete groups, the following is of interest.

Example 4. Suppose $\bar{B}$ is a torsion complete group of final rank $\aleph_{\alpha}$. For any positive integer $n$ we have,

$$
\mu\left(\bar{B}^{n}\right)= \begin{cases}n, & \text { if } n \leq \alpha \\ \infty, & \text { if } n>\alpha .\end{cases}
$$

To verify this assertion, observe first that we may ignore any bounded summand, so we assume that the rank of $\bar{B}$ is the same as the final rank. If $n>\alpha, \bar{B}^{n}$ is a $\oplus_{c}$ by [10, Theorem 5]. If $n \leq \alpha$, subgroups $X_{1}, \ldots, X_{n}$ of $\bar{B}$ can easily be constructed satisfying the conditions in Example 3. Then $A=X_{1} \cdots X_{n} \subseteq \bar{B}^{n}$, so $n \leq \mu\left(\bar{B}^{n}\right) \leq \mu(A)=n$, i.e., $\mu\left(\bar{B}^{n}\right)=n$.

This last example implies that $\mu\left(\bar{B}^{n}\right)$ depends only on the final rank of $\bar{B}$. This, in turn, clearly depends only on the size of the continuum, $c=2^{\aleph_{0}}$. In other words, the size of the continuum is to a certain extent determined by the Tor functor.

\section{REFERENCES}

1. D. Cutler and P. Dubois, Generalized final rank for arbitrary limit ordinals, Pacific J. Math. 37 (1971), 345-351.

2. P. Eklof, On singular compactness, Algebra Universalis 14 (1982), 310-316.

3. L. Fuchs, Infinite abelian groups, vol. 2, Academic Press, New York, 1973.

4. L. Fuchs and J. Irwin, On $p^{\omega+1}$-projective p-groups, Proc. London Math. Soc. 3 (1975), 459-470.

5. P. Griffith, Infinite abelian groups, Chicago Lectures in Math., Chicago and London, 1970.

6. P. Hill, The balance of Tor, Math. Z. 182 (1983), 179-188.

7. _ On the structure of abelian p-groups, Trans. Amer. Math. Soc. 288 (1985), 505-525. 
8. J. Irwin, T. Snabb, and T. Cellars, The torsion product of totally projective p-groups, Comment. Math. Univ. St. Paul 29 (1980), 1-5.

9. P. Keef, On the Tor functor and some classes of abelian groups, Pacific J. Math. 132 (1988), 63-84.

10. $\ldots$, On iterated torsion products of abelian p-groups, Rocky Mountain J. Math. (to appear).

11. __ S-groups, A-groups and the Tor functor, Comment. Math., Univ. St. Paul (to appear).

12. R. Nunke, Purity and subfunctors of the identity, Topics in Abelian Groups, Chicago, 1963, pp. 121-171.

13. $ـ$, On the structure of Tor, Proc. Colloq. Abelian Groups (Budapest, 1964), pp. 115124.

14. _ On the structure of Tor II, Pacific J. Math. 22 (1967), 453-467.

15. R. Warfield, $A$ classification theorem for abelian p-groups, Trans. Amer. Math. Soc. 37 (1973), 149-168.

16. B. Wick, The calculation of an invariant for Tor, Pacific J. Math. 112 (1984), 445-450.

Department of Mathematics, Whitman College, Walla Walla, Washington 99362 